\title{
Relationship between the pyroptosis of fibroblast-like synoviocytes and HMGB1 secretion in knee osteoarthritis
}

\author{
YANCHENG XIAO $^{1 *}$, LIANG DING $^{2 *}$, SONGJIANG YIN ${ }^{2}$, ZHENGQUAN HUANG $^{2}$, \\ $\mathrm{LI} \mathrm{ZHANG}^{2}$, WEI MEI ${ }^{2}$, PENG WU ${ }^{3}$, PEIMIN WANG ${ }^{2}$ and KE PAN ${ }^{1}$
}

\author{
${ }^{1}$ Department of Orthopedics, Liyang Hospital of Traditional Chinese Medicine, Liyang, Jiangsu 213300; \\ ${ }^{2}$ Department of Orthopedics, Jiangsu Province Hospital of Traditional Chinese Medicine, Affiliated Hospital of Nanjing \\ University of Chinese Medicine, Nanjing, Jiangsu 210029; ${ }^{3}$ Key Laboratory for Metabolic Diseases in Chinese Medicine, \\ First College of Clinical Medicine, Nanjing University of Chinese Medicine, Nanjing, Jiangsu 210023, P.R. China
}

Received November 10, 2019; Accepted September 11, 2020

DOI: $10.3892 / \mathrm{mmr} .2020 .11736$

\begin{abstract}
High mobility group box 1 (HMGB1) is an important downstream product of pyroptosis in macrophages, and it serves a vital role in numerous inflammatory diseases. Previous studies have reported that HMGB1 is released by fibroblast-like synoviocytes (FLSs) that are activated by inflammatory cytokines in knee osteoarthritis (KOA); however, the mechanism via which FLS promotes HMGB1 secretion in KOA remains unknown. According to our previous study, pyroptosis occurs in FLSs of patients with KOA and is mediated by Nod-like receptor protein (NLRP)1 or NLRP3 inflammasomes. However, the specific relationship between HMGB1 secretion and FLS pyroptosis requires further investigation. In the present study, the association between HMGB1 secretion and FLS pyroptosis was investigated in vitro and in vivo. In this study, western blotting, ELISA and reverse transcription-quantitative PCR were used to measure expression levels of proteins and mRNA. Caspase-1 activity assay
\end{abstract}

Correspondence to: Professor Peimin Wang, Department of Orthopedics, Jiangsu Province Hospital of Traditional Chinese Medicine, Affiliated Hospital of Nanjing University of Chinese Medicine, 155 Hanzhong Road, Nanjing, Jiangsu 210029, P.R. China E-mail: drwpm@163.com

Dr Ke Pan, Department of Orthopedics, Liyang Hospital of Traditional Chinese Medicine, 122 Xihou Road, Liyang, Jiangsu 213300, P.R. China

E-mail: pankedr@126.com

${ }^{*}$ Contributed equally

Abbreviations: KOA, knee osteoarthritis; FLS, fibroblast-like synoviocytes; HMGB1, high mobility group box 1; NLRP, Nod-like receptor protein; LPS, lipopolysaccharide; ASC, apoptosis-associated speck-like protein with a caspase-recruitment domain; GSDMD, gasdermin D; ACLT, anterior cruciate ligament resection

Key words: KOA, FLS, pyroptosis, HMGB1 and Hoechst 33342/PI double staining were used to observe the pyroptosis of FLSs. Hematoxylin and eosin staining was used to observe the destruction of cartilage in KOA. Increased expression levels of pyroptosis-related proteins and HMGB1 in the synovium of rat anterior cruciate ligament transection-induced KOA models were identified, and these changes were significantly mitigated via the intra-articular injection of a caspase-1 inhibitor. In vitro, FLSs were treated with lipopolysaccharide (LPS) + ATP to induce pyroptosis, and HMGB1 secretion was subsequently measured. LPS + ATP significantly increased the expression levels of pyroptosis-related proteins and HMGB1 in FLSs, and these effects were significantly mitigated by small interfering RNAs targeting NLRP1, NLRP3, apoptosis-associated speck-like protein with a caspase-recruitment domain or caspase-1. Therefore, the present results indicated that NLRP1/NLRP3 inflammasome-mediated and caspase-1-dependent FLS pyroptosis increased HMGB1 secretion in KOA. These findings may provide a therapeutic strategy to decrease synovial inflammatory responses during KOA progression.

\section{Introduction}

Knee osteoarthritis (KOA) is a common degenerative disease in the orthopedic field, which has a high disability rate. With the increasing aging of the social population, the incidence of KOA has increased to $45 \%$ in the last 20 years, and the incidence of KOA has exceeded $80 \%$ among people $>65$ years old (1). KOA is a chronic degenerative joint disease caused by multifactorial etiologies involving the loss of articular cartilage, subchondral bone sclerosis, cyst formation, osteophyte development, chronic pain, stiffness and lower extremity disability, which significantly affect the independence and quality of life of patients, primarily among elderly individuals (2).

Pyroptosis is a caspase-1-dependent form of programmed cell death that substantially differs from apoptosis, and involves canonical and non-canonical inflammasome activation pathways (3). Nod-like receptor protein (NLRP) inflammasomes, mainly the NLRP1 and NLRP3 inflammasomes, belong to a group of seven widely accepted inflammasome complexes: NLRP1, NLRP3, NLRC4, interferon $\gamma$ inducible protein 
16 , pyrin, caspase- 4 and absent in melanoma 2 (4). NLRPs directly interact with apoptosis-ssociated speck-like protein with a caspase-recruitment domain (ASC) to activate caspase-1 (cleaved caspase-1p10 and caspase-1p20), which in turn promotes gasdermin D (GSDMD) cleavage and translocation (5-7). This leads to the formation of a hole in the cell membrane that facilitates the release of intracellular inflammatory factors, such as IL-1 $\beta$ and IL-18 and ultimately results in cell death (8). Previous studies have reported that fibroblast-like synoviocytes (FLSs) undergo lipopolysaccharide (LPS) + ATP-induced pyroptosis in a caspase-1-dependent manner, mediated by activation of the NLRP1 and NLRP3 inflammasomes $(9,10)$.

High mobility group box 1 (HMGB1) was first identified as a nuclear protein that regulates transcription, replication and DNA repair (11). Extracellular HMGB1 has been identified as a crucial cytokine involved in the intestinal inflammatory response (12) and multiple inflammatory diseases, including osteoarthritis (13), rheumatoid arthritis (14), endotoxemia (15), epilepticus (16), stroke (17), diabetes (18), innate immunity, collagen disease, atherosclerosis, cancer and acute lung injury $(19,20)$. HMGB1 can be secreted by FLSs after stimulation with inflammatory factors in KOA (21-25), and HMGB1 levels in synovial tissues increase during KOA progression and correlate positively with KOA synovitis (26). HMGB1 can also stimulate chondrocytes to secrete MMPs, as well as promote cartilage autophagy and degeneration in the pathogenesis of KOA (21). Moreover, the HMGB1-LPS complex is reportedly transported into macrophages via RNA for advanced-glycation end products (RAGE)-dependent endocytosis, and intracellular LPS activates caspase-1 and leads to pyroptosis (27), indicating HMGB1 as an important factor in the inflammatory response in KOA. However, the specific mechanism via which FLSs promote HMGB1 secretion in KOA is yet to be elucidated. Notably, a previous study revealed that ASC-mediated caspase-1 activation can promote abnormal HMGB1 secretion during alveolar macrophage pyroptosis in acute lung injury (28). Therefore, the relationship between HMGB1 secretion and FLS pyroptosis in KOA requires further investigation.

The present study aimed to investigate the relationship between HMGB1 secretion and FLS pyroptosis in vivo and in vitro. Small interfering RNAs (siRNAs) and inhibitors were used to further elucidate the specific effect of FLS pyroptosis on HMGB1 secretion.

\section{Materials and methods}

Animals and samples. A total of 15 male Sprague-Dawley (SD) rats (Animal House Grant Certificate no. 201810A001; Beijing Vital River Laboratory Animal Technology Co., Ltd.; age, 2 months; weight, 200-300 g) were maintained in a specific pathogen-free laminar-flow housing apparatus, under controlled temperature $\left(25 \pm 2^{\circ} \mathrm{C}\right)$ and humidity $(60 \pm 5 \%)$ conditions with a 12-h light/dark cycle, all rats had free access to food and water. All animal protocols were approved by the Animal Care and Use Committee of the Nanjing University of Chinese Medicine. All experiments were conducted in accordance with the National Institutes of Health Guidelines for the Care and Use of Laboratory Animals (29).
The rats were randomly assigned to three groups: the normal group ( $n=5)$, the KOA group $(n=5)$ and the caspase-1 inhibitor group $(n=5)$. KOA was induced in the KOA and caspase-1 inhibitor groups via anterior cruciate ligament transection (ACLT) as previously described (30). Rats were anesthetized with an intraperitoneal injection of $30 \mathrm{mg} / \mathrm{kg}$ pentobarbital sodium (3\%). The joint capsule was cut along its medial side (normal group), or the anterior cruciate ligament was transected (KOA, caspase-1 inhibitor and HMGB1 inhibitor groups). The surgical results were examined via the front drawer test (31). Amikacin (Mutian Animal Pharmaceutical Co., Ltd.) was injected into the thigh of each rat at a dose of $10 \mathrm{mg} / \mathrm{kg}$ per day for 4 days after the operation. The rats were placed in cages allowing free movement after the operation, and the joints were not fixed. From day 14, the rats in the caspase-1 inhibitor group were given intra-articular injection of caspase-1 inhibitor VX765 (MedChemExpress, Inc.) at a dose of $50 \mathrm{mg} / \mathrm{kg}$ in $50 \mu \mathrm{l}$ sterilized physiological saline once a day for 2 weeks. Rats in the normal and KOA groups were given intra-articular injection of $50 \mu 1$ sterilized physiological saline. At day 28, all rats were sacrificed, and synovial tissues and cartilage were harvested. Synovial tissues were collected to detect protein and gene expression levels, as well as caspase-1 activity. Cartilage was subjected to hematoxylin and eosin (H\&E) staining to evaluate damage. Serum proteins were detected using specific ELISA kits.

FLS culture and treatment. Synovial tissues removed from five 2-month-old male SD rats were snipped into pieces of $\sim 3 \mathrm{~mm}^{3}$, homogenized in DMEM (Gibco; Thermo Fisher Scientific, Inc.) and incubated for $1 \mathrm{~h}$ at $37^{\circ} \mathrm{C}$ with $1 \mathrm{mg} / \mathrm{ml}$ type I collagenase (Sigma-Aldrich; Merck KGaA). The samples were filtered through a $100-\mu \mathrm{m}$ cell strainer. After dissociation, the FLSs were pelleted via centrifugation at $300 \mathrm{x}$ g at $\sim 25^{\circ} \mathrm{C}$ for $5 \mathrm{~min}$ and plated in DMEM supplemented with 10\% FBS (Gibco; Thermo Fisher Scientific, Inc.) and 1\% antibiotics (100 U/ml penicillin and $100 \mu \mathrm{g} / \mathrm{ml}$ streptomycin; Invitrogen; Thermo Fisher Scientific, Inc.). Cells were cultured at $37^{\circ} \mathrm{C}$ in a humidified atmosphere with $95 \%$ air and $5 \% \mathrm{CO}_{2}$, and were identified as described in our previous studies $(9,10)$. Primary FLSs from passages 3-5 were used for subsequent experiments.

To induce pyroptosis, FLSs were stimulated with LPS (3 $\mu \mathrm{g} / \mathrm{ml}$; Sigma-Aldrich; Merck KGaA) in DMEM for $12 \mathrm{~h}$ and then treated with ATP (Beijing Solarbio Science \& Technology Co., Ltd.) at $37^{\circ} \mathrm{C}(3 \mathrm{mM})$ for $4 \mathrm{~h}$. For the control group, FLSs were treated with DMEM for $12 \mathrm{~h}$ and then treated with DMEM in combination with $0.9 \%$ saline of the same volume as ATP for $4 \mathrm{~h}$. Compounds in the supernatant were detected using specific ELISA kits. FLSs were collected to detect protein and gene expression levels, and caspase-1 activity, as well as to perform immunofluorescence.

siRNA transfection. To inhibit NLRP1, NLRP3, ASC and caspase-1 mRNA expression levels in FLSs, commercially available specific siRNAs and control siRNAs (Guangzhou RiboBio Co., Ltd.) were used. The core nucleotide of siRNAs were as follows: NLRP1 siRNA, 5'-GGUGGAGCUGCAUCA CAUATT-dTdT-3' and Vehicle, 5'-UUCUCCGAACGUGUC ACGU-dTdT-3'; NLRP3 siRNA, 5'-GGAGAGACCUUUAUG AGAATT-dTdT-3' and Vehicle, 5'-UUCUCCGAACGUGUC 
Table I. Upstream primers and downstream primers for HMGB1 and GAPDH.

\begin{tabular}{lll}
\hline Gene & Primer & \\
\hline HMGB1 & Forward & Sequences $\left(5^{\prime} \rightarrow 3^{\prime}\right)$ \\
& Reverse & CTGCCTTCTCTTGTGACAAAGTGGAC \\
GAPDH & Forward & ACATACTCAGCACCAGCATCACC \\
& Reverse & CCTCCTTCGGCCTTCTTCTTGTTC \\
& TCATCCGCAGCAGTGTTGTTCC \\
\hline
\end{tabular}

HMGB1, high mobility group box 1 .

ACGU-dTdT-3'; ASC siRNA, 5'-CTGATAAACTCGTCA GCTA-dTdT-3' and Vehicle, 5'-UUCUCCGAACGUGUCACG U-dTdT-3'; and caspase-1 siRNA, 5'-GGGCAAGCCAGAUGU UUAU-dTdT-3' and Vehicle, 5'-UUCUCCGAACGUGUCACG U-dTdT-3'. The vehicles are the negative control siRNAs.

FLSs were plated in 6-well plates with the density of $1 \times 10^{4} / \mathrm{ml}$ and transfected with siRNAs using Lipofectamine ${ }^{\circledR}$ 2,000 (Invitrogen; Thermo Fisher Scientific, Inc.) according to the manufacturer's instructions. siRNAs were diluted in the transfection reagent and culture medium, and the FLSs in each plate were incubated with $20 \mathrm{pmol}$ siRNA at $37^{\circ} \mathrm{C}$ for $6 \mathrm{~h}$ before the addition of LPS + ATP to induce pyroptosis. To assess whether siRNA transfection was successful, the verification experiments were conducted independently before the formal transfection experiments. FLSs plated in 6-well plates with the density of $1 \times 10^{4} / \mathrm{ml}$ were divided into control, siRNA and vehicle groups.

Western blot analysis. Synovial tissues from individual rats were homogenized and lysed in RIPA lysis buffer (Beyotime Institute of Biotechnology). FLSs in different groups were also lysed in RIPA lysis buffer. Lysates were collected and centrifuged at $4^{\circ} \mathrm{C}$ at $12,000 \mathrm{x} \mathrm{g}$ for $10 \mathrm{~min}$, and protein concentrations were quantified with a BCA protein assay kit (Beyotime Institute of Biotechnology). Individual samples (20 $\mu \mathrm{g} /$ lane) were separated via $10 \%$ SDS-PAGE and were transferred onto PVDF membranes (Beyotime Institute of Biotechnology). The membranes were blocked with 5\% BSA (Beyotime Institute of Biotechnology) at $25^{\circ} \mathrm{C}$ for $2 \mathrm{~h}$ and then incubated overnight at $4^{\circ} \mathrm{C}$ with primary antibodies against the following proteins: Caspase-1p10 (1:1,000; cat. no. sc-56036; Santa Cruz Biotechnology, Inc.), NLRP3 (1:500; cat. no. ab214185; Abcam), NLRP1 (1:1,000; cat. no. sc-166368; Santa Cruz Biotechnology, Inc.), pro-IL-1 $\beta$ (1:2,000; cat. no. ab9722; Abcam), IL-1 $\beta$ (1:1,000; cat. no. ab9722; Abcam), GSDMD-C (1:500; cat. no. sc-393581; Santa Cruz Biotechnology, Inc.), HMGB1 (1:5,000; cat. no. ab79823; Abcam) and $\beta$-actin (1:2,000; cat. no. 60008-1-Ig; ProteinTech Group, Inc.). The membranes were then incubated with horseradish peroxidase-conjugated Affinipure goat anti-rabbit $\operatorname{IgG}$ $(\mathrm{H}+\mathrm{L})$ (1:20,000; cat. no. SA00001-2; ProteinTech Group, Inc.) for $2 \mathrm{~h}$ at room temperature. The bands were visualized using an enhanced chemiluminescent (Thermo Fisher Scientific, Inc.) method, and the grayscale values of the protein bands (average gray value) were quantified with Photoshop CS5 (Adobe Systems, Inc.) using $\beta$-actin as an internal reference: Target protein gray value/internal reference gray value.
Reverse transcription-quantitative PCR (RT-qPCR). Total RNA was extracted from synovial tissues and FLSs using an RNA-Quick Purification kit (ES Science Biotech Co., Ltd.; www.esunbio.com). RNA concentration and purity were measured with a spectrophotometer, and the target $260 \mathrm{~nm} / 280 \mathrm{~nm}$ ratio was between 1.8 and 2.0. RT was performed using a HiScriptQ RT SuperMix kit (cat. no. R222-01; Vazyme Biotech Co., Ltd.) according to the manufacturer's instructions (stage $1,1 \mathrm{rep}, 50^{\circ} \mathrm{C}, 15 \mathrm{~min}$; stage $2,1 \mathrm{rep},-80^{\circ} \mathrm{C}, 5 \mathrm{sec}$ ). RNA expression was measured with a ChamQ SYBR qPCR Master Mix kit (cat. no. Q331; Vazyme Biotech Co., Ltd.) and an ABI 7500 real-time PCR system (Applied Biosystems; Thermo Fisher Scientific, Inc.) according to the manufacturer's instructions (stage $1,1 \mathrm{rep}, 95^{\circ} \mathrm{C}, 30 \mathrm{sec}$; stage $2,40 \mathrm{reps}, 95^{\circ} \mathrm{C}, 10 \mathrm{sec}, 60^{\circ} \mathrm{C}, 30 \mathrm{sec}$; and stage $3,1 \mathrm{rep}$, $\left.95^{\circ} \mathrm{C}, 15 \mathrm{sec}, 60^{\circ} \mathrm{C}, 60 \mathrm{sec}, 95^{\circ} \mathrm{C}, 15 \mathrm{sec}\right)$. GAPDH was used as an internal reference. All the primer sequences are provided in Table I. All reactions were performed in triplicate, and the results were analyzed using the $2^{-\Delta \Delta C \mathrm{Cq}}$ method (32). The experiment was repeated $\geq 3$ times.

Caspase-1 activity assay. To investigate pyroptosis, caspase-1 activity in individual samples was determined based on p-nitroaniline (pNA) production using a colorimetric assay kit (cat. no. C1101; Beyotime Institute of Biotechnology). Briefly, after detecting the caspase-1 protein concentration in individual cellular and synovial tissue samples, the samples were diluted to $1-3 \mathrm{mg} / \mathrm{ml}$. Triplicate aliquots of each sample $(10 \mu \mathrm{l})$ were incubated with $10 \mu 1$ Ac-YVAD-pNA $(2 \mathrm{mM})$ in 96-well microplates at $37^{\circ} \mathrm{C}$ for $100 \mathrm{~min}$, and caspase- 1 activity in individual wells was measured based on the absorbance at $405 \mathrm{~nm}$ using a Multimode plate reader (PerkinElmer, Inc.).

Hoechst 33342/PI double staining. Hoechst 33342/PI double staining was used for morphological analysis. FLSs were plated in replicates at $1 \times 10^{6}$ cells per well in 6 -well plates. The cells were collected via centrifugation at $300 \mathrm{x}$ g for $5 \mathrm{~min}$ $\sim 25^{\circ} \mathrm{C}$, suspended in cell staining buffer, incubated with $5 \mu \mathrm{l}$ Hoechst 33342 and $5 \mu$ PI solution (cat. no. CA1120; Beijing Solarbio Science \& Technology Co., Ltd.) at $4^{\circ} \mathrm{C}$ for $30 \mathrm{~min}$ and observed under a fluorescence microscope (magnification, x200; Leica Microsystems GmbH).

ELISA. HMGB1 and IL-1 $\beta$ levels in serum and culture supernatants were assessed using specific ELISA kits (HMGB1. cat. no. F15640; IL-1ß. cat. no. F15810; Westang Biotechnology Co., Ltd.) according to the manufacturer's instructions. The 

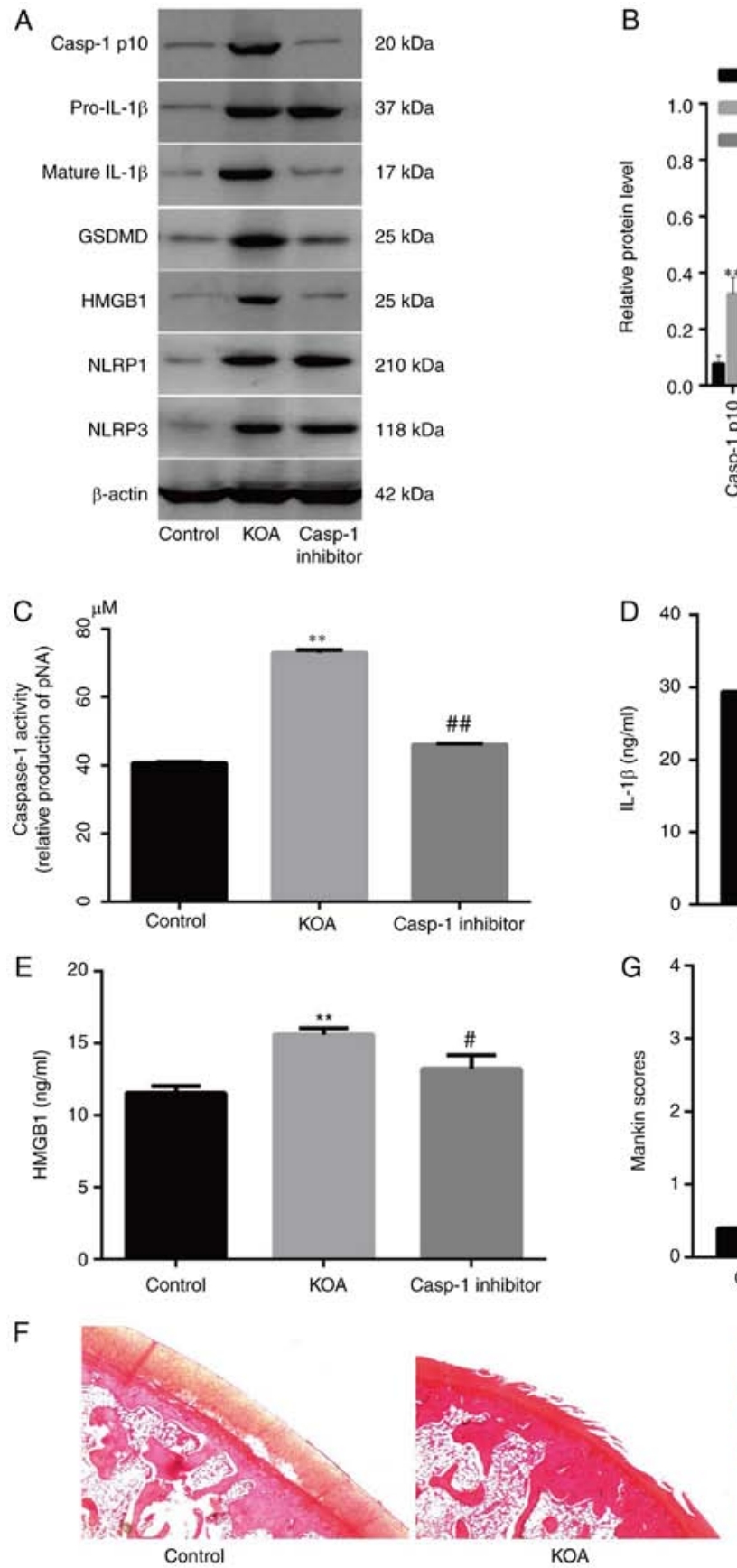
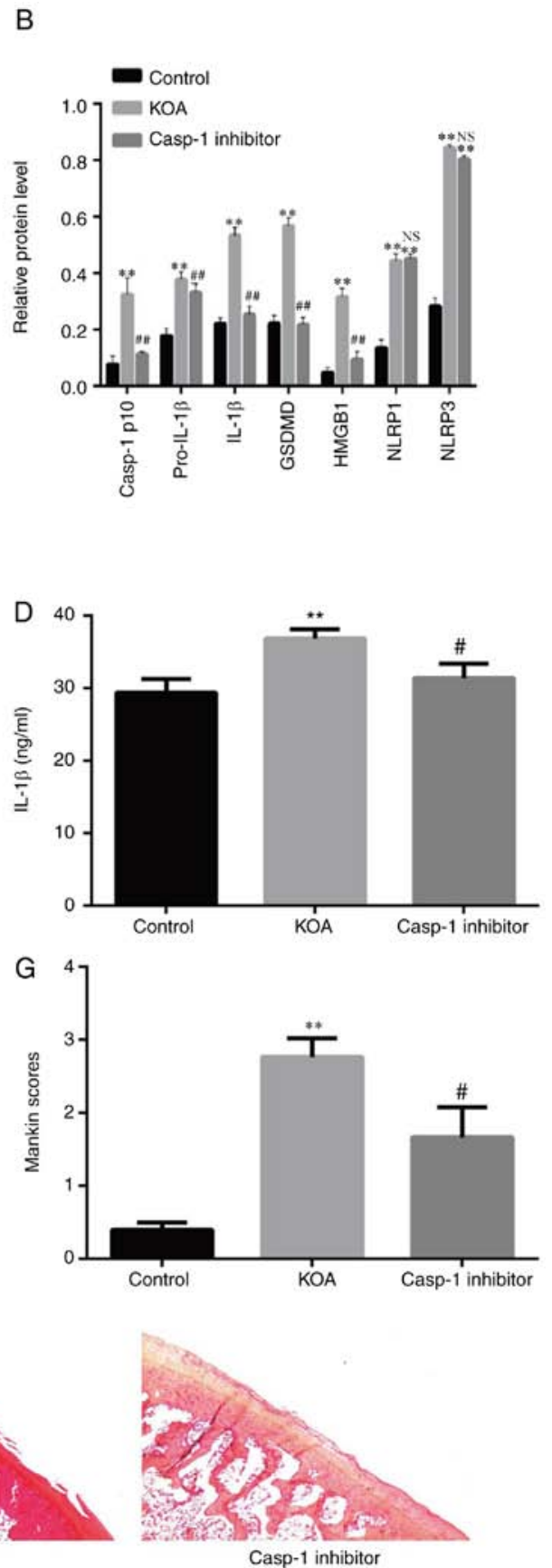

Figure 1. Anterior cruciate ligament resection-induced pyroptosis increases HMGB1 secretion. (A) Western blotting results of the (B) relative expression levels of pyroptosis-related proteins and HMGB1. (C) Casp-1 activity in synovial tissues. Levels of (D) HMGB1 and (E) IL-1 $\beta$ in serum. (F) Hematoxylin and eosin staining for cartilage tissue (magnification, $\mathrm{x} 200$ ) with (G) relative Mankin scores from five rats. Data are presented as the mean \pm SD of each group ( $\mathrm{n}=3$ per group) from three separate experiments. ${ }^{* *} \mathrm{P}<0.01$ vs. control group; ${ }^{\#} \mathrm{P}<0.05,{ }^{\# \#} \mathrm{P}<0.01$ vs. KOA group; NS vs. KOA group. HMGB1, high mobility group box 1 ; NLRP, Nod-ike receptor protein; KOA, knee osteoarthritis; GSDMD, gasdermin D; casp-1, caspase-1; NS, not significant.

blood ( $6 \mathrm{ml}$ per rat) was collected from the abdominal aorta of rats and the serum sample was collected via centrifugation at $1,000 \mathrm{x} \mathrm{g}$ for $15 \mathrm{~min}$ at $4^{\circ} \mathrm{C}$. Experimental and control samples were tested simultaneously in triplicate.

Histopathological analysis. Tissue was collected from rats and fixed with $4 \%$ paraformaldehyde at $25^{\circ} \mathrm{C}$ for $72 \mathrm{~h}$, soaked in $15 \%$ EDTA at $25^{\circ} \mathrm{C}$ for 12 weeks for decalcification, then tissue was dehydrated in alcohol for $2 \mathrm{~h}$. The dehydrated tissue was treated with xylene solution for dehydration and then was paraffin-embedded. Paraffin-embedded tissue was cut into sections (thickness, $5 \mu \mathrm{m}$ ). Then, paraffin-embedded sections were treated with xylene solution for $30 \mathrm{~min}$ for dewaxing. Tissue sections were treated with gradient concentration of ethanol (100, 90, 80, 70 and 65\%; 2 min per concentration) for rehydration. The sections were then stained in hematoxylin solution at $25^{\circ} \mathrm{C}$ for $3-5 \mathrm{~min}$ and rinsed with running water for $30 \mathrm{sec}$, then stained sections were divided with hydrochloric acid for $30 \mathrm{sec}$ and rinsed again with running water for $15 \mathrm{~min}$. Stained sections were stained in eosin solution at $25^{\circ} \mathrm{C}$ for 2-3 min after dehydration in alcohol with the concentration of 70 and $90 \%$ for $10 \mathrm{~min}$. Then, the stained sections were 

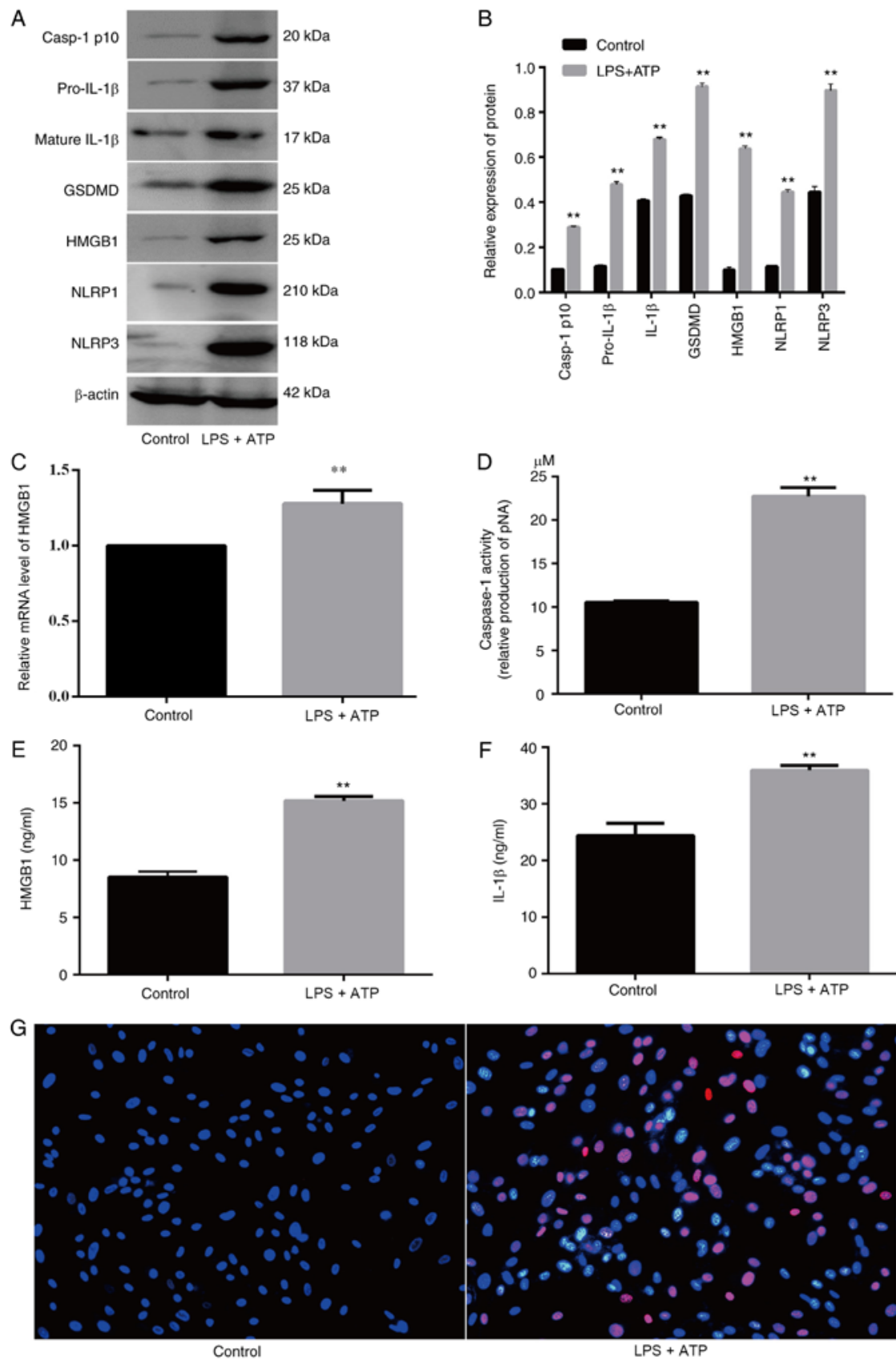

Figure 2.LPS + ATP-induced FLS pyroptosis increases HMGB1 secretion. (A) Western blotting results of the (B) relative expression levels of pyroptosis-related proteins and HMGB1. (C) Relative mRNA expression of HMGB1. (D) Casp-1 activity in FLSs. Levels of (E) HMGB1 and (F) IL-1 $\beta$ in supernatant. (G) FLS pyroptosis was observed via Hoechest 33342/PI double staining (magnification, $\mathrm{x} 200$ ), dead nuclei were stained pink or bright red. Data are presented as the mean \pm SD of each group ( $n=3$ per group) from three separate experiments. ${ }^{* *} \mathrm{P}<0.01$ vs. control group. HMGB1, high mobility group box 1 ; NLRP, Nod-like receptor protein; KOA, knee osteoarthritis; GSDMD, gasdermin D; casp-1, caspase-1; LPS, lipopolysaccharide.

placed into xylene solution for 20 min. Finally, an appropriate amount of neutral gum was added, the glass was covered and sealed. All the stained sections were observed under optical electron microscope (magnification, x200). The degree of KOA in individual rats was assessed by observing cartilage destruction using the Mankin's scoring system ( 0 , normal; 1 , mild; 2 , moderate; 3 , severe).

Statistical analysis. Each experiment was repeated three times. Statistical analysis was performed using GraphPad Prism 6.0 Software (GraphPad Software, Inc.). Data are presented as the mean \pm SD. Group comparisons were assessed with the one-way ANOVA with Bonferroni's post hoc test or Student's t-test, or two-way ANOVA with Bonferroni's post hoc test for comparison of multiple columns. $\mathrm{P}<0.05$ was considered to indicate a statistically significant difference.

\section{Results}

ACLT-induced pyroptosis increases HMGB1 secretion in vivo. ACLT significantly increased the expression levels of pyroptosis-associated proteins (NLRP1, NLRP3, GSDMD, IL-1 $\beta$, pro-IL-1 $\beta$ and caspase-1 p10; Fig. 1A, B and D) and HMGB1 (Fig. 1A and $\mathrm{E}$ ) in synovial tissue and serum; however, these 

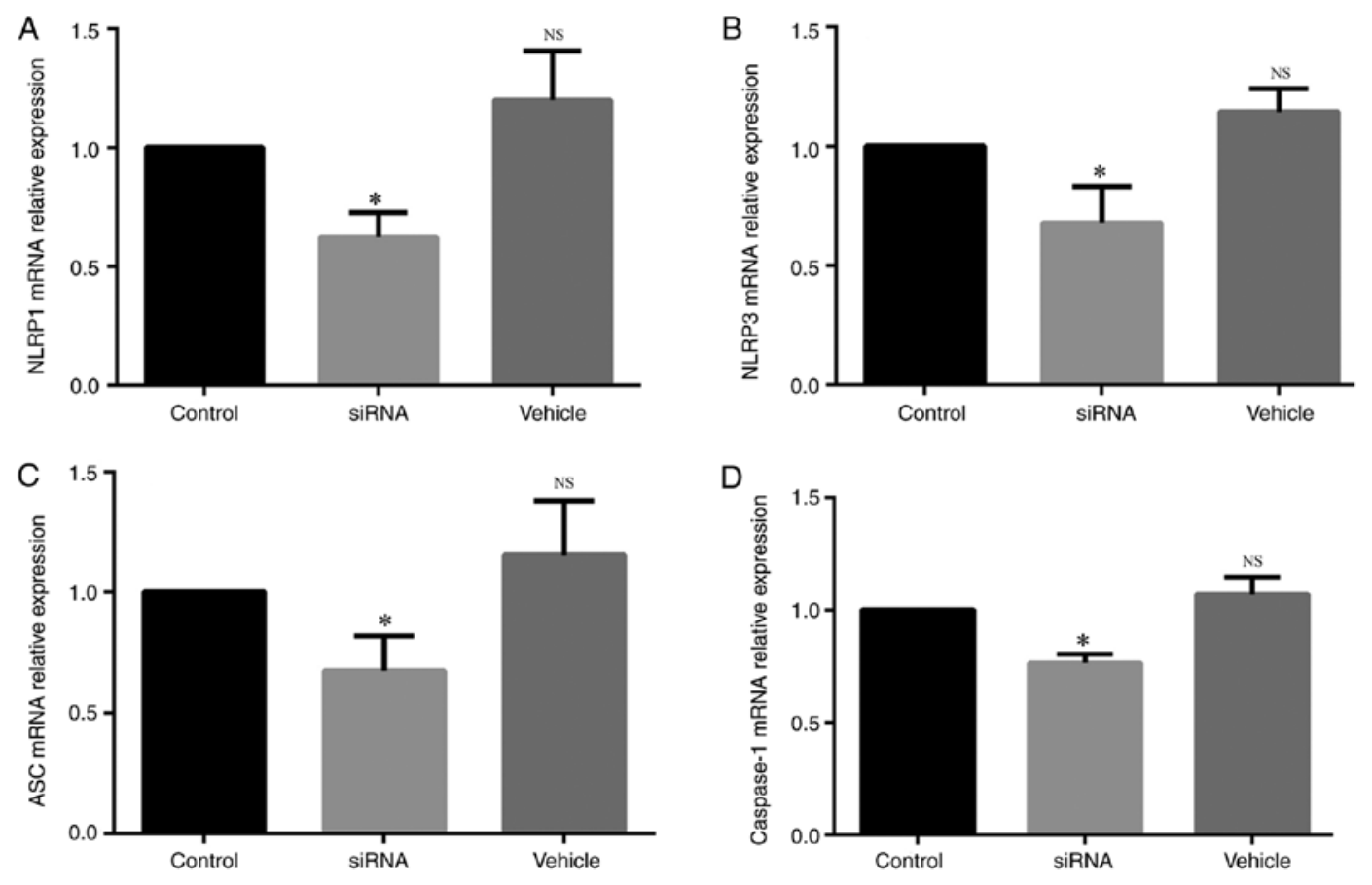

Figure 3. siRNA transfection decreases mRNA expression. Relative mRNA expression levels of (A) NLRP1, (B) NLRP3, (C) ASC and (D) caspase-1. Data are presented as the mean $\pm \mathrm{SD}$ of each group ( $\mathrm{n}=3$ per group) from three separate experiments. ${ }^{*} \mathrm{P}<0.05$ vs. control group and vehicle group; NS vs. control group. NLRP, Nod-like receptor protein; siRNA, small interfering RNA; ASC, apoptosis-associated speck-like protein with a caspase-recruitment domain.

effects were attenuated by a caspase-1 inhibitor, which also decreased caspase-1 activity in synovial tissue (Fig. 1C). It was identified that cartilage degeneration occurred in ACLT-induced inflammatory arthritis and was markedly inhibited by the caspase-1 inhibitor (Fig. 1F).

LPS + ATP-induced FLS pyroptosis increases HMGBI secretion in vitro. LPS + ATP significantly induced FLS pyroptosis, which was accompanied by increased caspase-1 activity (Fig. 2D) and upregulated expression levels of pyroptosis-related proteins (NLRP1, NLRP3, GSDMD, IL-1 $\beta$, pro-IL-1 $\beta$ and caspase-1 p10; Fig. 2A, B and F). The FLSs with pyroptosis were stained pink (Fig. 2G). Moreover, LPS + ATP significantly increased HMGB1 levels (Fig. 2A-C and E).

siRNA transfection decreases $m R N A$ expression in vitro. To observe whether the siRNA transfection was successful, the different siRNAs were used to knockdown the mRNA expression levels of NLRP1, NLRP3, ASC and caspase-1. The mRNA expression of NLRP1 was significantly lower in the siRNA group compared with the control group (Fig. 3A), and the same results were identified for the mRNA expression levels of NLRP3 (Fig. 3B), ASC (Fig. 3C) and caspase-1 (Fig. 3D).

NLRPI knockdown attenuates HMGB1 secretion in vitro. Our previous study revealed that NLRP1 inflammasomes are important in the pathogenesis of KOA as they induce FLS pyroptosis (9). To observe the specific effect of NLRP1 on HMGB1 secretion, FLSs were exposed to a NLRP1-specific siRNA or the control. HMGB1 protein expression was significantly higher in the LPS + ATP and vehicle groups compared with the NLRP1 siRNA and control groups (Fig. 4A and B), and similar results were obtained for relative HMGB1 mRNA expression (Fig. 4C) and HMGB1 levels in the supernatant (Fig. 4D)

NLRP3 knockdown attenuates HMGB1 secretion in vitro. It was previously proved that NLRP3 inflammasomes are involved in the pathogenesis of KOA by inducing FLS pyroptosis (9). Thus, NLRP3-specific siRNA was used to assess the effect of NLRP3 on HMGB1 secretion. Healthy FLSs served as the control group. HMGB1 protein expression (Fig. 5A and B) was significantly higher in the LPS + ATP and vehicle groups compared with in the NLRP3 siRNA and control groups. Similar results were obtained for relative HMGB1 RNA expression (Fig. 5C) and HMGB1 levels in the supernatant (Fig. 5D).

ASC knockdown mitigates FLS pyroptosis and reduces HMGB1 secretion in vitro. ASC is an important component of inflammasomes (33). To examine whether ASC knockdown affects LPS + ATP-induced FLS pyroptosis and HMGB1 secretion, FLSs were exposed to an ASC-specific siRNA or a control. The expression levels of pyroptosis-related proteins and HMGB1 (Fig. 6A and B) were significantly higher in the LPS + ATP and vehicle groups compared with the ASC siRNA and control groups, and the relative RNA expression of HMGB1 demonstrated the same pattern (Fig. 6C). Similar results were obtained for HMGB1 and IL-1 $\beta$ levels in the supernatant of the FLSs in different groups (Fig. 6D and E). Therefore, ASC may be important for FLS pyroptosis and HMGB1 secretion.

Caspase-1 knockdown decreases HMGB1 secretion in vivo. To further verify the correlation between FLS pyroptosis and HMGB1 secretion, caspase-1-specific siRNA was used to inhibit FLS pyroptosis, and untreated FLSs served as the 
A
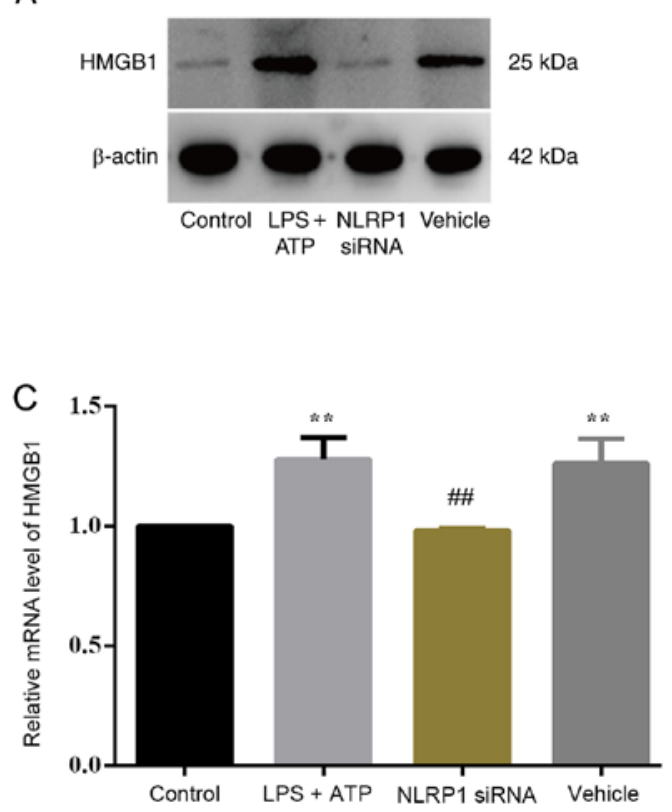

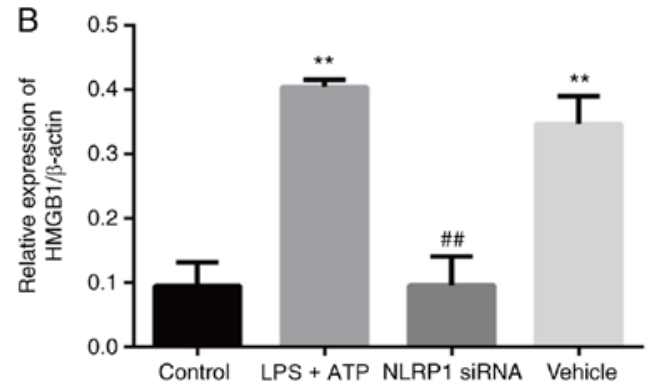

D

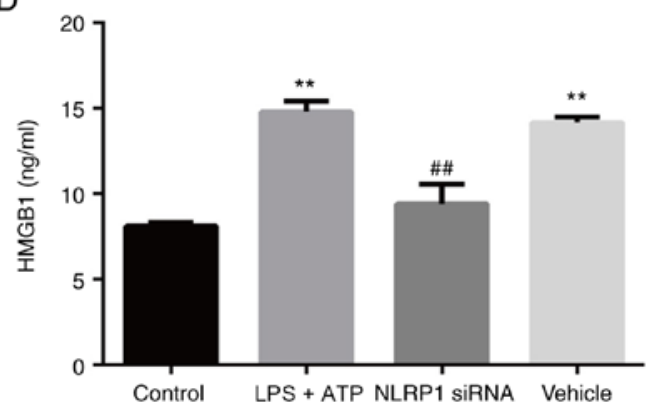

Figure 4. Knockdown of NLRP1 by a specific siRNA mitigates HMGB1 secretion. (A) Western blotting results of the (B) relative protein expression of HMGB1. (C) Transcription level of HMGB1. (D) Levels of HMGB1 in supernatant. Data are presented as the mean \pm SD of each group ( $\mathrm{n}=3$ per group) from three separate experiments. ${ }^{* *} \mathrm{P}<0.01$ vs. control group; ${ }^{\# \#} \mathrm{P}<0.01$ vs. LPS + ATP group. HMGB1, high mobility group box 1; NLRP, Nod-like receptor protein; siRNA, small interfering RNA; LPS, lipopolysaccharide.

A

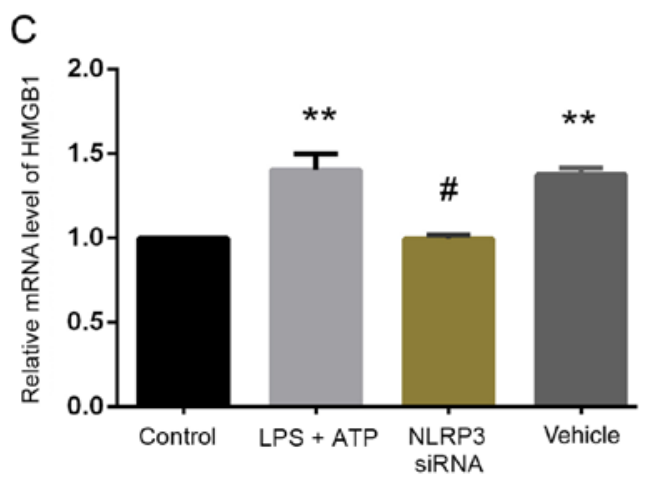

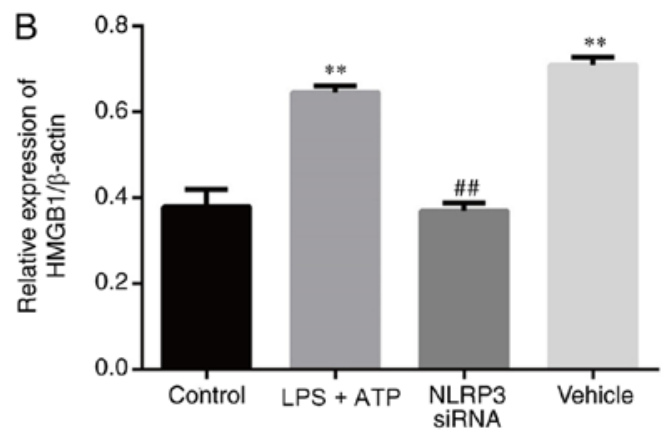

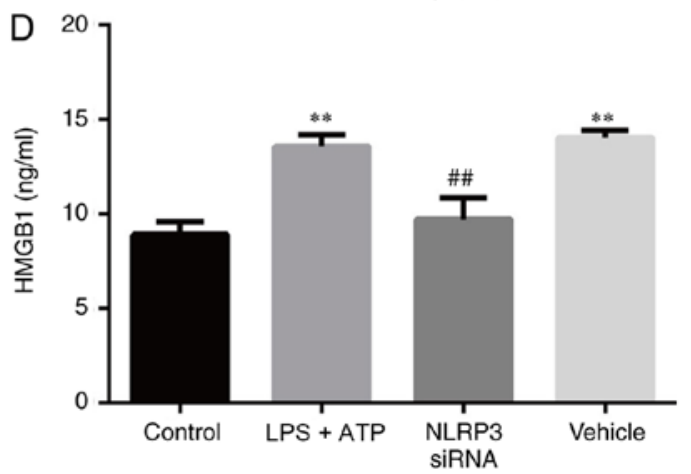

Figure 5. Knockdown of NLRP3 by specific siRNA mitigates HMGB1 expression. (A) Western blotting results of the (B) relative protein expression of HMGB1. (C) Relative mRNA expression of HMGB1. (D) Levels of HMGB1 in supernatant. Data are presented as the mean \pm SD of each group ( $\mathrm{n}=3$ per group) from three separate experiments. ${ }^{* *} \mathrm{P}<0.01$ vs. control group; ${ }^{\mathrm{P}} \mathrm{P}<0.05$ and ${ }^{\# \#} \mathrm{P}<0.01$ vs. LPS + ATP group. HMGB1, high mobility group box $1 ;$ NLRP, Nod-like receptor protein; siRNA, small interfering RNA; LPS, lipopolysaccharide.

control group. HMGB1 protein expression was significantly increased in the LPS + ATP and vehicle groups compared with the caspase-1 siRNA and control groups (Fig. 7A and B). Moreover, similar results were obtained for relative HMGB1 RNA expression (Fig. 7C) and HMGB1 levels in the supernatant (Fig. 7D).

\section{Discussion}

Synovitis is common in KOA, and most patients with KOA with obvious clinical symptoms have synovitis $(34,35)$. Inflammatory reactions in synovial tissues are accompanied by congestion, edema, the accumulation of inflammatory 

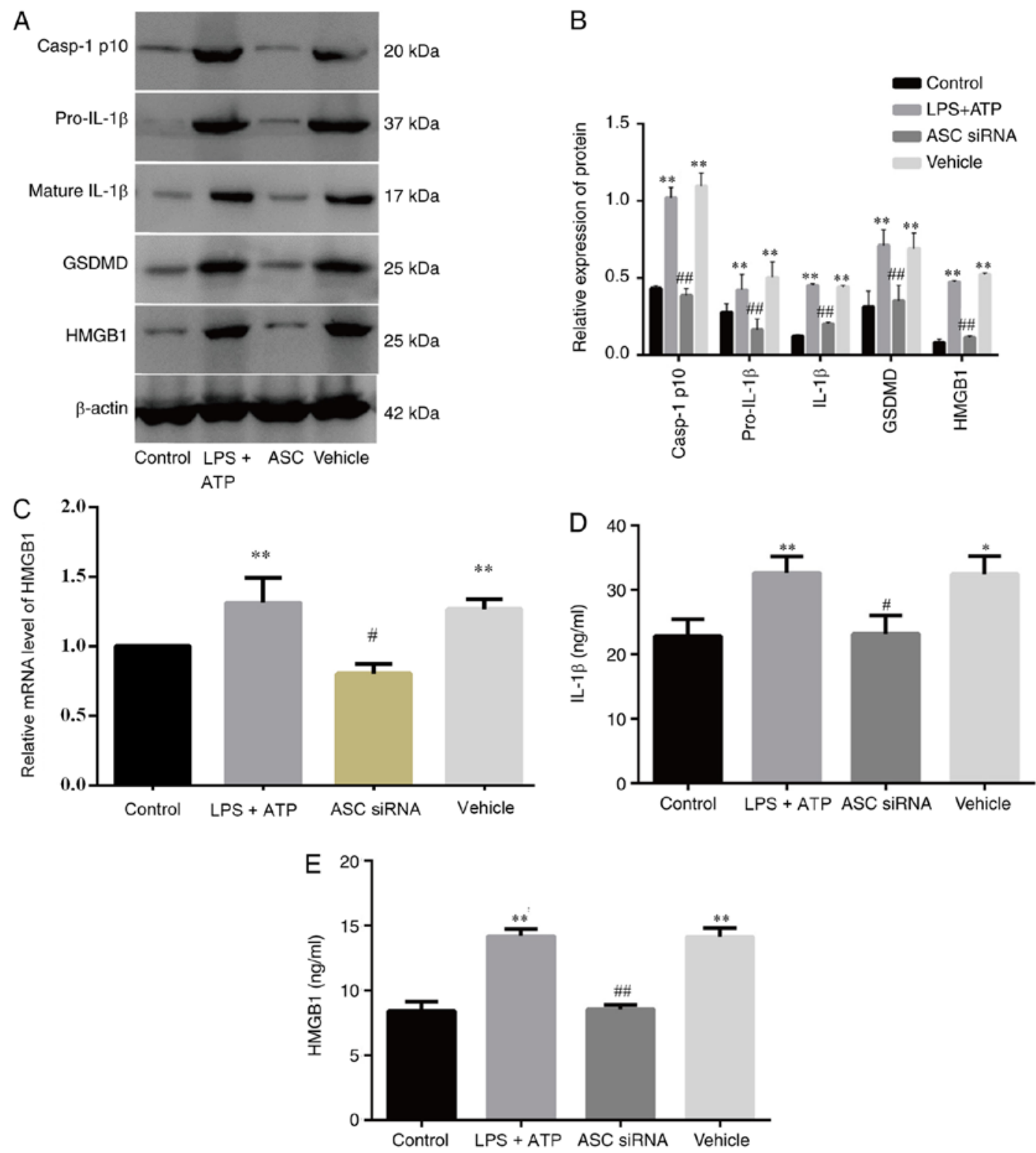

Figure 6. Knockdown of ASC by specific siRNA mitigates fibroblast-like synoviocyte pyroptosis and decreases HMGB1 secretion. (A) Western blotting results of the (B) relative expression levels of pyroptosis-related proteins and HMGB1. (C) HMGB1 gene expression. Levels of (D) HMGB1 and (E) IL-1 $\beta$ in cell supernatant. Data are presented as the mean $\pm \mathrm{SD}$ of each group $\left(\mathrm{n}=3\right.$ per group) from three separate experiments. ${ }^{*} \mathrm{P}<0.05,{ }^{*} \mathrm{P}<0.01$ vs. control group; ${ }^{\sharp} \mathrm{P}<0.05$, ${ }^{\# \#} \mathrm{P}<0.01$ vs. LPS + ATP group. HMGB1, high mobility group box 1; siRNA, small interfering RNA; LPS, lipopolysaccharide; ASC, apoptosis-associated speck-like protein with a caspase-recruitment domain; GSDMD, gasdermin D; casp-1, caspase-1.

mediators and the secretion of various types of proteases, ultimately resulting in abnormal cartilage metabolism $(36,37)$. FLSs are important factors in KOA inflammation and joint destruction during the pathological progression of KOA, primarily by secreting a wide range of proinflammatory mediators, such as IL-1 $\beta$, IL-18, TNF- $\alpha$ and MMPs (38).

HMGB1 has been reported to serve a proinflammatory role in $\operatorname{KOA}(23,26)$, and HMGB1 levels in synovial fluid are closely associated with the severity of KOA (39). On the one hand, as an important proinflammatory factor in KOA, HMGB1 is responsible for promoting cartilage degradation and inducing synovitis by binding to RAGE, Toll-like receptor 4 and other receptor (40). On the other hand, HMGB1 in complex with LPS or IL-1 enhances the production of proinflammatory cytokines in OA FLSs (41). These findings indicate that HMGB1 is an important factor in KOA pathogenesis and that HMGB1 levels are highly associated with synovitis in KOA. While the specific mechanism of HMGB1 production in KOA is unknown, HMGB1 is found to be secreted by FLSs stimulated with inflammatory factors in KOA $(42,43)$. Moreover, whether HMGB1 release is involved in FLS pyroptosis in KOA is yet to be fully elucidated. Therefore, the present study investigated the specific association between HMGB1 secretion and FLS pyroptosis.

NLRP1 and NLRP3 expression levels are significantly higher in patients with KOA (44). The NLRP1 and NLRP3 inflammasomes are key regulators in the innate immune system that promote the release of downstream caspase-1-dependent proinflammatory cytokines, such as IL-1 $\beta$ and IL-18 (45), suggesting that these inflammasomes are important mediators of FLS pyroptosis. Furthermore, inflammasomes mediate pyroptosis by activating caspase-1 (46). In 
A
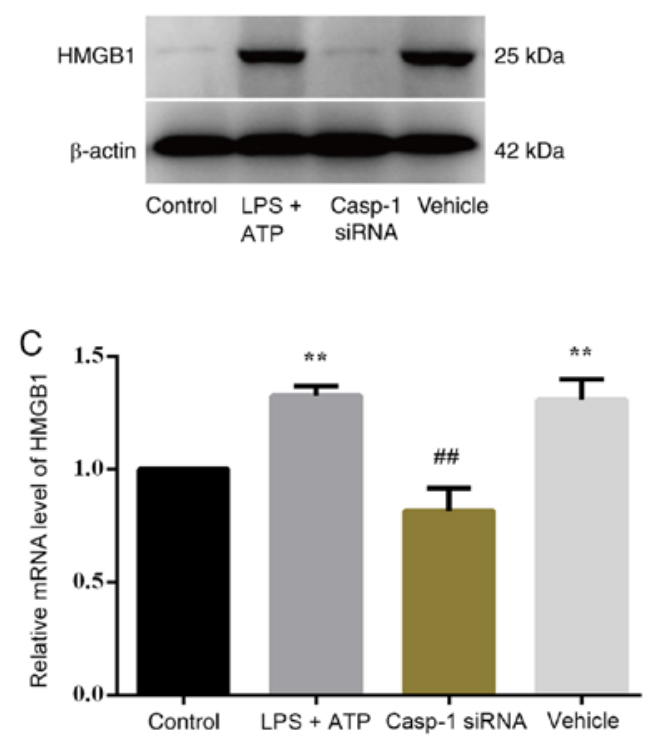
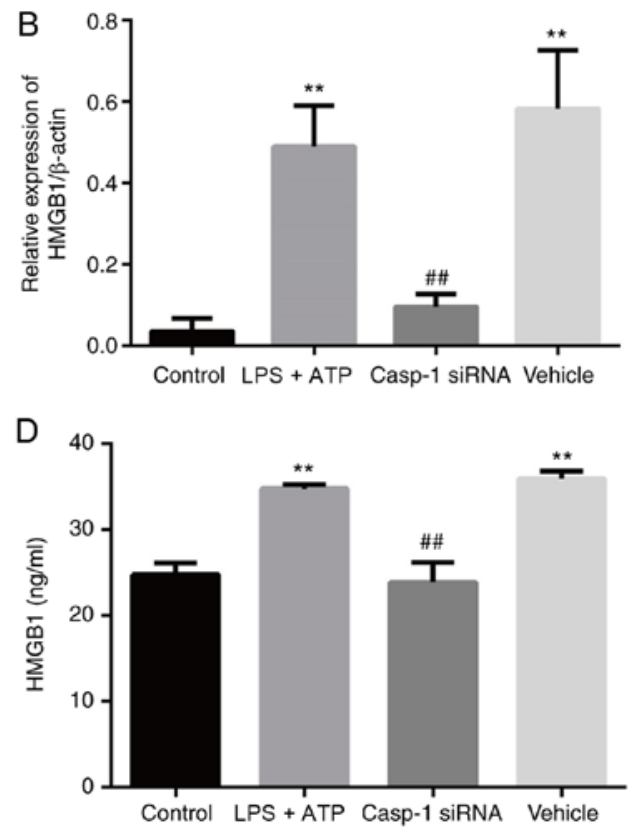

Figure 7. Knockdown of caspase-1 by specific siRNA mitigates HMGB1 secretion. (A) Western blotting results of the (B) relative protein expression of HMGB1. (C) Gene expression of HMGB1. (D) Levels of HMGB1 in the cell supernatant. Data are presented as the mean \pm SD of each group ( $\mathrm{n}=3$ per group) from three separate experiments. ${ }^{* *} \mathrm{P}<0.01$ vs. control group; ${ }^{\# \#} \mathrm{P}<0.01$ vs. LPS + ATP group. HMGB1, High Mobility Group Box 1; siRNA, small interfering RNA; LPS, lipopolysaccharide; ASC, apoptosis-associated speck-like protein with a caspase-recruitment domain; casp-1, caspase-1.

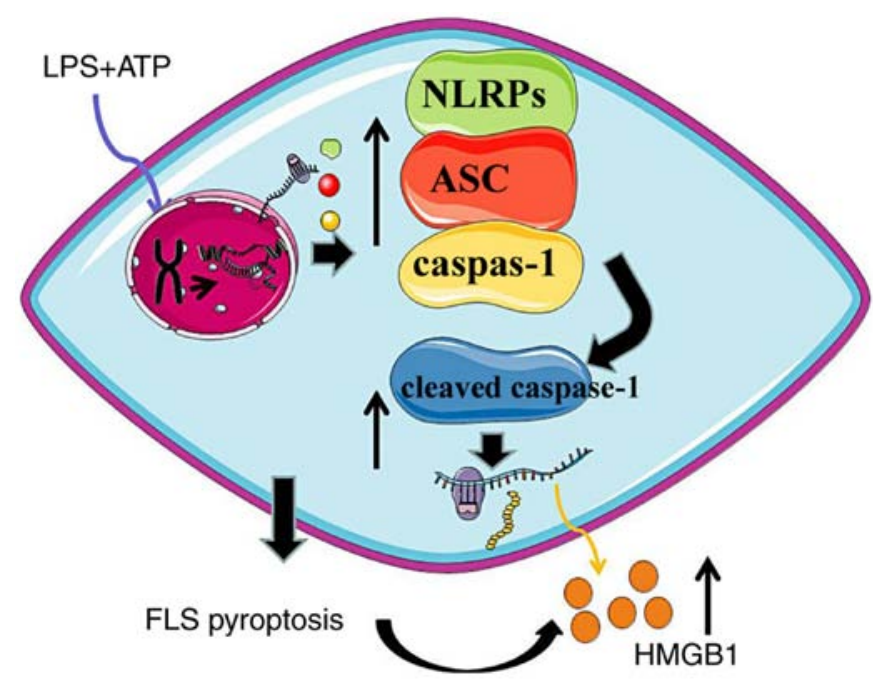

Figure 8. Pyroptosis enhances HMGB1 secretion in FLSs. Present results suggested that HMGB1 secretion was closely associated with the inflammasome-mediated and caspase-1-dependent FLS pyroptosis. ASC, apoptosis-associated speck-like protein with a caspase-recruitment domain; NLRP, Nod-like receptor protein; FLS, fibroblast-like synoviocyte; LPS, lipopolysaccharide; HMGB1, high mobility group box 1.

the present study, in vivo experiments in a rat ACLT-induced KOA model were conducted, and increased levels of HMGB1 and pyroptosis-related proteins were found in synovial tissues, which were significantly decreased by treatment with a caspase-1 inhibitor. Therefore, it was suggested that there was an association between HMGB1 secretion and pyroptosis in synovial tissue. To further demonstrate the association between HMGB1 secretion and pyroptosis, LPS + ATP was used to induce FLS pyroptosis in vitro. Increased caspase-1 activity and pyroptosis-related protein expression levels indicated FLS pyroptosis. It was also identified that HMGB1 secretion by FLSs was mediated via NLRP1 and NLRP3 inflammasomes and caspase-1 activity. Knockdown of NLRP1, NLRP3 or caspase-1 attenuated HMGB1 secretion by FLSs, suggesting that FLS pyroptosis may be responsible for HMGB1 secretion by FLSs in KOA.

ASC is important for the formation of the NLRP3 and NLRP1 inflammasomes in inflammatory diseases (47), suggesting that ASC is an important component of pyroptosis. A previous study reported that transfection of alveolar macrophages with ASC-specific siRNA decreased ASC expression and mitigated pyroptosis (28). In the present study, it was demonstrated that ASC was an important factor in FLS pyroptosis, as transfection with ASC-specific siRNA not only inhibited caspase-1-dependent FLS pyroptosis, but also decreased HMGB1 secretion. The aforementioned experimental results indicated that HMGB1 production was closely associated with caspase-1-dependent FLS pyroptosis induced by NLRP1 and NLRP3 inflammasomes and ASC expression. Hence, it was considered that HMGB1 secretion may be closely associated with the function of activated caspase-1 (Fig. 8). HMGB1 has been reported to increase IL-1 $\beta$ production via the NLRP3 inflammasome in vascular and liver injury $(48,49)$, suggesting that HMGB1 may be a pyroptosis induction factor, but it remains unknown whether HMGB1 has the same function in KOA. The present results indicated that pyroptosis was an important factor in HMGB1 secretion, but the specific effect of HMGB1 on pyroptosis in synovial cells requires further investigation.

In conclusion, the present findings provide a novel insight into the role of HMGB1 secretion in KOA pathogenesis. The current in vivo and in vitro experiments demonstrated that NLRP1 and NLRP3 inflammasome-mediated FLS pyroptosis 
enhanced HMGB1 secretion. Thus, decreasing FLS pyroptosis by inhibiting the activation of the NLRP1 and NLRP3 inflammasomes and caspase-1 may be an effective strategy for treating KOA.

\section{Acknowledgements}

Not applicable.

\section{Funding}

The present study was supported by The National Natural Science Foundation of China (grant no. 8187151765) and the Jiangsu provincial Leading Talents Program of Traditional Chinese Medicine of China (grant no. SLJ0207).

\section{Availability of data and materials}

The datasets used and/or analyzed during the current study are available from the corresponding author on reasonable request.

\section{Authors' contributions}

YX mainly participated in the conceptualization, experiment operation and writing-original draft of the study. LD mainly participated in the conceptualization and experiment operation. SY mainly participated in experiment operation. ZH was involved in the data analysis and methodolody. LZ participated in purchasing the experiment materials, the resource administration and the data analysis. WM wrote the original draft, contributed to the validation of the study and data analysis. $\mathrm{PWu}$ was involved in formal analysis of the study, data analysis and image processing. PWa mainly participated in editing the manuscript, modifying the draft, analysis and interpretation of data, as well as purchasing and feeding of rats. KP participated in the conceptualization, supervision of the study and modification of draft, and analysis and interpretation of data. All authors read and approved the final manuscript.

\section{Ethics approval and consent to participate}

All animal protocols were approved by the Animal Care and Use Committee of the Nanjing University of Chinese Medicine.

\section{Patient consent for publication}

Not applicable.

\section{Competing interests}

All the authors declare that they have no competing interests.

\section{References}

1. Johnson VL and Hunter DJ: The epidemiology of osteoarthritis. Best Pract Res Clin Rheumatol 28: 5-15, 2014.

2. Gong Z, Liu R, Yu W, Wong TK, Guo Y and Sun Y: Acutherapy for knee osteoarthritis relief in the elderly: A systematic review and meta-analysis. Evid Based Complement Alternat Med 2019: $1868107,2019$.
3. Xia X, Wang X, Zheng Y, Jiang J and Hu J: What role does pyroptosis play in microbial infection? J Cell Physiol 234: 7885-7892, 2019.

4. Platnich JM and Muruve DA: NOD-like receptors and inflammasomes: A review of their canonical and non-canonical signaling pathways. Arch Biochem Biophys 670: 4-14, 2019.

5. Bergsbaken T, Fink SL and Cookson BT: Pyroptosis: Host cell death and inflammation. Nat Rev Microbiol 7: 99-109, 2009.

6. Schroder K, Muruve DA and Tschopp J: Innate immunity: Cytoplasmic DNA sensing by the AIM2 inflammasome. Curr Biol 19: R262-R265, 2009.

7. Duprez L, Wirawan E, Vanden Berghe T and Vandenabeele P: Major cell death pathways at a glance. Microbes Infect 11: 1050-1062, 2009

8. Dong W, Zhu Q, Yang B, Qin Q, Wang Y, Xia X, Zhu X, Liu Z, Song E and Song Y: Polychlorinated biphenyl quinone induces caspase 1-mediated pyroptosis through induction of pro-inflammatory HMGB1-TLR4-NLRP3-GSDMD signal axis. Chem Res Toxicol 32: 1051-1057, 2019.

9. Zhao LR, Xing RL, Wang PM, Zhang NS, Yin SJ, Li XC and Zhang L: NLRP1 and NLRP3 inflammasomes mediate LPS/ATP-induced pyroptosis in knee osteoarthritis. Mol Med Rep 17: 5463-5469, 2018.

10. Zhang L, Zhang L, Huang Z, Xing R, Li X, Yin S, Mao J, Zhang N, Mei W, Ding L and Wang P: Increased HIF-1 $\alpha$ in knee osteoarthritis aggravate synovial fibrosis via fibroblast-like synoviocyte pyroptosis. Oxid Med Cell Longev 2019: 6326517, 2019.

11. Venereau E, De Leo F, Mezzapelle R, Careccia G, Musco G and Bianchi ME: HMGB1 as biomarker and drug target. Pharmacol Res 111: 534-544, 2016

12. Burzynski LC and Clarke M: Death is coming and the clot thickens, as pyroptosis feeds the fire. Immunity 50: 1339-1341, 2019.

13. Biscetti F, Flex A, Pecorini G, Angelini F, Arena V, Stigliano E, Gremese E, Tolusso B and Ferraccioli G: The role of high-mobility group box protein 1 in collagen antibody-induced arthritis is dependent on vascular endothelial growth factor. Clin Exp Immunol 184: 62-72, 2016

14. Chung H, Hong SJ, Choi SW, Koo JY, Kim M, Kim HJ, Park SB and Park CG: High mobility group box 1 secretion blockade results in the reduction of early pancreatic islet graft loss. Biochem Biophys Res Commun 514: 1081-1086, 2019.

15. Magna M and Pisetsky DS: The role of HMGB1 in the pathogenesis of inflammatory and autoimmune diseases. Mol Med 20: 138-146, 2014.

16. Wang FC, Pei JX, Zhu J, Zhou NJ, Liu DS, Xiong HF, Liu XQ, Lin DJ and Xie Y: Overexpression of HMGB1 A-box reduced lipopolysaccharide-induced intestinal inflammation via HMGB1/TLR4 signaling in vitro. World J Gastroenterol 21: 7764-7776, 2015.

17. Yu S, Zhang H, Hei Y, Yi X, Baskys A, Liu W and Long Q: High mobility group box-1 (HMGB1) antagonist BoxA suppresses status epilepticus-induced neuroinflammatory responses associated with Toll-like receptor $2 / 4$ down-regulation in rats. Brain Res 1717: 44-51, 2019.

18. Tian X, Liu C, Shu Z and Chen G: Review: Therapeutic targeting of HMGB1 in stroke. Curr Drug Deliv 14: 785-790, 2017.

19. Paudel YN, Angelopoulou E, Piperi C, Balasubramaniam V, Othman I and Shaikh MF: Enlightening the role of high mobility group box 1 (HMGB1) in inflammation: Updates on receptor signalling. Eur J Pharmacol 858: 172487, 2019.

20. Vijayakumar EC, Bhatt LK and Prabhavalkar KS: High mobility group box-1 (HMGB1): A potential target in therapeutics. Curr Drug Targets 20: 1474-1485, 2019.

21. Lin SS, Yuan LJ, Niu CC, Tu YK, Yang CY and Ueng SWN: Hyperbaric oxygen inhibits the HMGB1/RAGE signaling pathway by upregulating Mir-107 expression in human osteoarthritic chondrocytes. Osteoarthritis Cartilage 27: 1372-1381, 2019.

22. Wagner G, Lehmann C, Bode C, Miosge N and Schubert A: High mobility group box 1 protein in osteoarthritic knee tissue and chondrogenic progenitor cells: An ex vivo and in vitro study. Cartilage: Mar 26, 2019 (Online ahead of print).

23. Taniguchi N, Kawakami Y, Maruyama I and Lotz M: HMGB proteins and arthritis. Hum Cell 31: 1-9, 2018.

24. Alsousi AA and Igwe OJ: Redox-active trace metal-induced release of high mobility group box 1(HMGB1) and inflammatory cytokines in fibroblast-like synovial cells is Toll-like receptor 4 (TLR4) dependent. Biochim Biophys Acta Mol Basis Dis 1864: 3847-3858, 2018 
25. Nefla M, Holzinger D, Berenbaum F and Jacques C: The danger from within: Alarmins in arthritis. Nat Rev Rheumatol 12: 669-683, 2016.

26. Ke X, Jin G, Yang Y, Cao X, Fang R, Feng X and Lei B: Synovial fluid HMGB-1 levels are associated with osteoarthritis severity. Clin Lab 61: 809-818, 2015.

27. Kim HM and Kim YM: HMGB1: LPS delivery vehicle for caspase-11-mediated pyroptosis. Immunity 49: 582-584, 2018.

28. Hou L, Yang Z, Wang Z, Zhang X, Zhao Y, Yang H, Zheng B Tian W, Wang S, He Z and Wang X: NLRP3/ASC-mediated alveolar macrophage pyroptosis enhances HMGB1 secretion in acute lung injury induced by cardiopulmonary bypass. Lab Invest 98: 1052-1064, 2018.

29. National Research Council (US) Institute for Laboratory Animal Research. Guide for the Care and Use of Laboratory Animals. Washington (DC), National Academies Press (US), 1996. Available from: https://www.ncbi.nlm.nih.gov/books/ NBK232589/ doi: 10.17226/5140

30. Xing R, Wang P, Zhao L, Xu B, Zhang N and Li X: Mechanism of TRPA1 and TRPV4 participating in mechanical hyperalgesia of rat experimental knee osteoarthritis. Arch Rheumatol 32: 96-104, 2017

31. Miao EA, Leaf IA, Treuting PM, Mao DP, Dors M, Sarkar A, Warren SE, Wewers MD and Aderem A: Caspase-1-induced pyroptosis is an innate immune effector mechanism against intracellular bacteria. Nat Immunol 11: 1136-1142, 2010.

32. Schmittgen TD and Livak KJ: Analyzing real-time PCR data by the comparative CT method. Nat Protoc 3: 1101-1108, 2008.

33. Martel-Pelletier J, Raynauld JP, Mineau F, Abram F, Paiement P, Delorme P and Pelletier JP: Levels of serum biomarkers from a two-year multicentre trial are associated with treatment response on knee osteoarthritis cartilage loss as assessed by magnetic resonance imaging: An exploratory study. Arthritis Res Ther 19: 169, 2017.

34. Samuelsson K, Magnussen RA, Alentorn-Geli E, Krupic F Spindler KP, Johansson C, Forssblad $\mathbf{M}$ and Karlsson $\mathbf{J}$ : Equivalent knee injury and osteoarthritis outcome scores 12 and 24 months after anterior cruciate ligament reconstruction: Results from the swedish national knee ligament register. Am J Sports Med 45: 2085-2091, 2017

35. Steinhaus ME, Christ AB and Cross MB: Total knee arthroplasty for knee osteoarthritis: Support for a foregone conclusion? HSS J 13: 207-210, 2017.

36. Kong R, Gao J, Si Y and Zhao D: Combination of circulating miR-19b-3p, miR-122-5p and miR-486-5p expressions correlates with risk and disease severity of knee osteoarthritis. Am J Transl Res 9: 2852-2864, 2017.

37. Zhang L, Xing R, Huang Z, Zhang N, Zhang L, Li X and Wang P. Inhibition of synovial macrophage pyroptosis alleviates synovitis and fibrosis in knee osteoarthritis. Mediators Inflamm 2019 : 2165918,2019
38. Zhang C, Yu W, Huang C, Ding Q, Liang C, Wang L, Hou Z and Zhang Z: Chrysin protects human osteoarthritis chondrocytes by inhibiting inflammatory mediator expression via HMGB1 suppression. Mol Med Rep 19: 1222-1229, 2019.

39. Shu Z, Miao X, Tang T, Zhan P, Zeng L and Jiang $Y$ : The GSK-3 $\beta / \beta$-catenin signaling pathway is involved in HMGB1-induced chondrocyte apoptosis and cartilage matrix degradation. Int J Mol Med 45: 769-778, 2020.

40. Wähämaa H, Schierbeck H, Hreggvidsdottir HS, Palmblad K, Aveberger AC, Andersson U and Harris HE: High mobility group box protein 1 in complex with lipopolysaccharide or IL-1 promotes an increased inflammatory phenotype in synovial fibroblasts. Arthritis Res Ther 13: R136, 2011.

41. Garcia-Arnandis I, Guillen MI, Gomar F, Pelletier JP, Martel-Pelletier J and Alcaraz MJ: High mobility group box 1 potentiates the pro-inflammatory effects of interleukin-1 $\beta$ in osteoarthritic synoviocytes. Arthritis Res Ther 12: R165, 2010.

42. Loeser RF, Goldring SR, Scanzello CR and Goldring MB Osteoarthritis: A disease of the joint as an organ. Arthritis Rheum 64: 1697-1707, 2012.

43. Terada C, Yoshida A, Nasu Y, Mori S, Tomono Y, Tanaka M, Takahashi HK, Nishibori M, Ozaki T and Nishida K: Gene expression and localization of high-mobility group box chromosomal protein-1 (HMGB-1)in human osteoarthritic cartilage. Acta Med Okayama 65: 369-377, 2011.

44. Fan C, Zhao X, Guo X, Cao X and Cai J: P2X4 promotes interleukin-1 $\beta$ production in osteoarthritis via NLRP1. Mol Med Rep 9: 340-344, 2014

45. Harapas CR, Steiner A, Davidson S and Masters SL: An update on autoinflammatory diseases: Inflammasomopathies. Curr Rheumatol Rep 20: 40, 2018

46. Kesavardhana S and Kanneganti TD: Mechanisms governing inflammasome activation, assembly and pyroptosis induction. Int Immunol 29: 201-210, 2017.

47. Zhang B, Zhang Y, Xu T, Yin Y, Huang R, Wang Y, Zhang J, Huang D and Li W: Chronic dexamethasone treatment results in hippocampal neurons injury due to activate NLRP1 inflammasome in vitro. Int Immunopharmacol 49: 222-230, 2017.

48. Kim EJ, Park SY, Baek SE, Jang MA, Lee WS, Bae SS, Kim K and Kim CD: HMGB1 Increases IL-1 $\beta$ production in vascular smooth muscle cells via NLRP3 inflammasome. Front Physiol 9: 313, 2018.

49. Geng Y, Ma Q, Liu YN, Peng N, Yuan FF, Li XG, Li M, Wu YS, Li BL, Song WB, et al: Heatstroke induces liver injury via IL-1 $\beta$ and HMGB1-induced pyroptosis. J Hepatol 63: 622-633, 2015.

This work is licensed under a Creative Commons Attribution-NonCommercial-NoDerivatives 4.0 International (CC BY-NC-ND 4.0) License. 